Check for updates

Cite this: RSC Adv., 2017, 7, 25298

Received 6th March 2017

Accepted 1st May 2017

DOI: $10.1039 / c 7 r a 02734 f$

rsc.li/rsc-advances

\section{Benchmark characterization of the thermoelectric properties of individual single-crystalline CdS nanowires by a $\mathrm{H}$-type sensor $\dagger$}

\author{
Haidong Wang, (D) $\ddagger^{a}$ Dingshan Zheng, $\dot{t}^{\mathrm{b}}$ Xing Zhang, ${ }^{\star c}$ Hiroshi Takamatsu (D) *a \\ and Weida $\mathrm{Hu}^{\star b}$
}

A precision $\mathrm{H}$-type sensor method has been developed to measure the thermoelectric performance of individual single-crystalline CdS nanowires for the first time. A nanomanipulation probe was used to directly pick up an individual nanowire from the array and place it on the sensor. Our method is generally applicable to any nanowire synthesized in either array or powder form. By simply changing the external electrical circuits, the Seebeck coefficient, thermal conductivity, and electrical conductivity have been measured on the same nanowire sample to ensure high accuracy and reliability. CdS nanowires have a large Seebeck coefficient over $300 \mu \mathrm{V} \mathrm{K}-1$ due to their wide band gap, while their thermal conductivity is only one-tenth of that of the bulk material owing to the significant phonon-surface scattering. The figure of merit, $Z T$, of the CdS nanowire is 0.01 at $320 \mathrm{~K}$, which is larger by two orders of magnitude than the value for $\mathrm{Bi}_{2} \mathrm{~S}_{3}$ nanowire, showing a trend of rapid increase above $300 \mathrm{~K}$.

\section{Introduction}

The rapidly increasing global demand for clean energy harvesting has led to an intense interest in thermoelectric materials that interconvert heat and electrical energy without the use of a heat engine or any moving parts. ${ }^{1-3}$ However, applications of thermoelectrics are still quite limited due to their low energy conversion efficiency. ${ }^{4,5}$ According to the dimensionless figure of merit of thermoelectric materials, $Z T=S^{2} \sigma T / \lambda$, where $S, T, \sigma$, and $\lambda$ are the Seebeck coefficient, temperature, electrical conductivity, and thermal conductivity, respectively, a high thermoelectric efficiency can be achieved for a material with large $S$, high $\sigma$, and low $\lambda$. However, maximizing $Z T$ is quite challenging because $S, \sigma$, and $\lambda$ are coupled to each other so that an increase in one parameter often has an adverse effect on another parameter., ${ }^{4,6}$ In recent years, a significant breakthrough has been made in maximizing $Z T$ by reducing the geometry of the sample from three-dimensional bulk structures to one-dimensional nanowires. ${ }^{7-9}$ Meanwhile, a variety of

\footnotetext{
${ }^{a}$ Department of Mechanical Engineering, Kyushu University, Fukuoka 819-0395, Japan. E-mail: takamatsu@mech.kyushu-u.ac.jp

${ }^{b}$ National Lab for Infrared Physics, Shanghai Institute of Technical Physics, Chinese Academy of Sciences, Shanghai 200083, People's Republic of China. E-mail: wdhu@ mail.sitp.ac.cn

'Department of Engineering Mechanics, Tsinghua University, Beijing 100084, People's Republic of China. E-mail: x-zhang@tsinghua.edu.cn

$\dagger$ Electronic supplementary information (ESI) available: Synthesis method of CdS nanowires, SEM images of CdS nanowire and EBID process, temperature response of H-type sensor and 2D thermal analysis result. See DOI: 10.1039/c7ra02734f

$\ddagger$ Equally contributed authors.
}

nanostructures such as superlattices, ${ }^{9}$ nanocomposites,${ }^{10}$ and quantum dots ${ }^{11}$ have been created to increase $Z T$. The physical mechanisms can be understood based on two features. First, low-dimensional materials exhibit sharp peaks in the density of electronic states, indicating high thermopower. ${ }^{12-14}$ Second, the characteristic size of a low-dimensional material can be one or more orders of magnitude smaller than the mean free path of the phonons but is larger than that of the electrons and holes. ${ }^{7}$ Because phonons are the main heat carriers for most semiconductors, it is possible to reduce the thermal conductivity of the nanostructured system while preserving its bulk-like electronic structures. ${ }^{7}$

Evaluation of the thermoelectric performance of an individual nanowire is important and essential for understanding the basic mechanisms and for the development of efficient thermoelectric devices. However, this is quite challenging because of the two following problems: (1) the thermoelectric properties of a nanowire are highly sensitive to its geometric size, crystalline structure, doping level, etc., with significant differences between the individual nanomaterials. ${ }^{15}$ The best strategy is to measure all three parameters involved in the $Z T$ formula, namely the Seebeck coefficient, and electrical and thermal conductivities, using the same individual nanowire. Unfortunately, currently the method that meets this purpose is quite limited.6,15 (2) Sample preparation is difficult for the chemical vapor deposition (CVD) grown nanowire arrays. The nanowires in arrays cannot be separated and picked up by dispersing the samples on a copper grid. The nanowires are easily contaminated or damaged during the common ultrasonic dispersion process. It is therefore considerably difficult to 
suspend an individual nanowire for characterization of its thermoelectric parameters.

The method that has a potential to be used for measuring the thermoelectric properties of individual nanowire is only that uses suspended microdevices. ${ }^{16,17}$ While the four-terminal sensing technique greatly improves the measurement accuracy, the micro-fabrication process is quite complicated. In the previous work, we used a T-type sensor method to measure the thermoelectric properties of an individual $\mathrm{Bi}_{2} \mathrm{~S}_{3}$ nanowire. ${ }^{15}$ The length, width, and thickness of the suspended nanofilm sensor were about $10 \mu \mathrm{m}, 600 \mathrm{~nm}$, and $40 \mathrm{~nm}$, respectively, much smaller than the geometric size of the commonly used microdevices. This was the reason why the suspended nanofilm sensor had an ultra-high thermal sensitivity. For measuring the thermoelectric power, however, two sensors that respectively work as a Joule heater and a thermometer are needed. ${ }^{6}$ The Ttype method had only one sensor and thus it made us use a complex AC heating/DC detecting scheme. ${ }^{15}$ In addition, our previous method is not applicable to nanowires grown in arrays, but only to those in the powder form.

In this report, we have developed the method from the T-type sensor to the H-type sensor, where a nanowire sample bridges two suspended nanofilm sensors, forming a letter " $H$ ". The measurement accuracy and reliability have been much improved. Using the H-type sensor, the thermoelectric performance of an individual single-crystalline cadmium sulfide (CdS) nanowire is reported for the first time. CdS nanowires have attracted intense attention for use in transistors, solar cells, photo detectors, light-emitting diodes, thermoelectric materials, and other applications because of their direct wide band gap of $2.4 \mathrm{eV}^{18-20}$ However, to our knowledge, no thermoelectric properties has been reported on the CdS nanowires. Here, we picked up a single CdS nanowire directly from the arrays using a nanomanipulation probe, and measured the intrinsic properties of as-grown CdS nanowire without contamination or damage.

\section{Experiment}

High quality single-crystalline CdS nanowire arrays were synthesized on a silicon substrate using the solid-source catalytic CVD method. ${ }^{21}$ More details about the synthesis of the CdS nanowires can be found in the ESI. $\dagger$ The optical and scanning electron microscope (SEM) images of the CdS nanowire arrays are shown in Fig. 1.

By using different synthesis methods, semiconductor nanowires can be prepared in different forms such as powders, bundles, and arrays. To prepare the nanowires in the powder form, an ultrasonic dispersion method is usually used to separate a single nanowire on a copper grid. However, this method is not applicable for the nanowire arrays because the nanowire can be easily contaminated or damaged during the dispersion process. Here we successfully picked up an individual CdS nanowire from the arrays by using a microprobe as shown in Fig. 1(d). The nanowire was attached to the probe tip by the Coulomb force. This is a direct and non-destructive method that can be applied to any semiconductor nanowire array. Additional SEM images are presented in the ESI. $\dagger$
Fig. 2 shows the high-resolution transmission electron microscope (TEM) image, selected area electron diffraction (SAED) pattern, and energy dispersive X-ray (EDX) spectrum of the prepared CdS nanowire. The results clearly confirm the pure $\mathrm{Cd}$ and $\mathrm{S}$ chemical composition and the high quality singlecrystalline structure. The observed lattice spacing is $0.322 \mathrm{~nm}$, corresponding to the (002) plane. In the nanowire length direction vertical to the (002) plane, the layered crystal structure suppresses the heat conduction and leads to lower thermal conductivity. In contrast to the bulk material, the phonon scattering at the surface of the nanowire is greatly enhanced and is expected to significantly decrease the thermal conductivity.

Fig. 3(a) shows the top SEM image of the suspended H-type sensor with the CdS nanowire. In the beginning, two nanofilm sensors $(8 \mathrm{~nm} \mathrm{Ti} / 40 \mathrm{~nm} \mathrm{Pt})$ and an electrode pad were fabricated by using the standard electron beam lithography and physical vapor deposition methods. Then, the sensors were completely suspended after the removal of the $\mathrm{SiO}_{2}$ layer by buffered hydrofluoric acid and etching of the silicon by $\mathrm{CF}_{4}$ plasma. The etched depth was about $1.2 \mu \mathrm{m}$. Additional SEM images and details regarding the fabrication process can be found in the ESI. $\dagger$

Next, we picked up an individual CdS nanowire (Fig. 1(d)) and placed it on the H-type sensor using a tungsten microprobe installed on a nanomanipulator from Kleindiek Nanotechnik. To reduce the electrical and thermal contact resistances between the nanowire and the sensor/electrode pad, Pt was deposited at four contact points using the electron beam induced deposition (EBID) method. All nanomanipulations, EBID processing, and thermoelectric measurements were carried out in a FEI Versa 3D ${ }^{\mathrm{TM}}$ dual-beam system (see ESI $\dagger$ ).

Fig. 3(b-d) show three measurement schemes for the thermal conductivity $\lambda$, electrical conductivity $\sigma$, and Seebeck coefficient $S$ of the same nanowire, respectively. In the $\lambda$ measurement, two sensors were used as the Joule heater and the precise resistance thermometer, respectively. The electrical resistance of each sensor was measured by using two highprecision digital multimeters (Keithley 2002). The temperature rise of the sensor was linearly proportional to its resistance change. The temperature coefficient of the resistance and the thermal conductivity of the sensor were carefully calibrated beforehand. A detailed description of the calibration process can be found in ref. 22. In the experiment, a relatively large temperature rise $(\sim 18 \mathrm{~K})$ was generated at the sensor used as the heater. Due to the heat conduction through the nanowire, a small temperature rise was measured at the other sensor that was larger for the nanowires with the higher thermal conductivity values. $\lambda$ of the individual CdS nanowire was extracted from the results of $2 \mathrm{D}$ thermal analysis (see the ESI $\dagger$ ). In contrast to our previous T-type method, the H-type sensor shows a much higher thermal sensitivity because the temperature response of one sensor can be detected simultaneously with the electrical heating of the other sensor. Meanwhile, the estimated temperature resolution of the nanofilm sensor was less than $0.01 \mathrm{~K}^{23}$ The $\mathrm{H}$-type sensor provides a suitable approach for the measurement of a thermoelectric nanomaterial with a low thermal conductivity. 

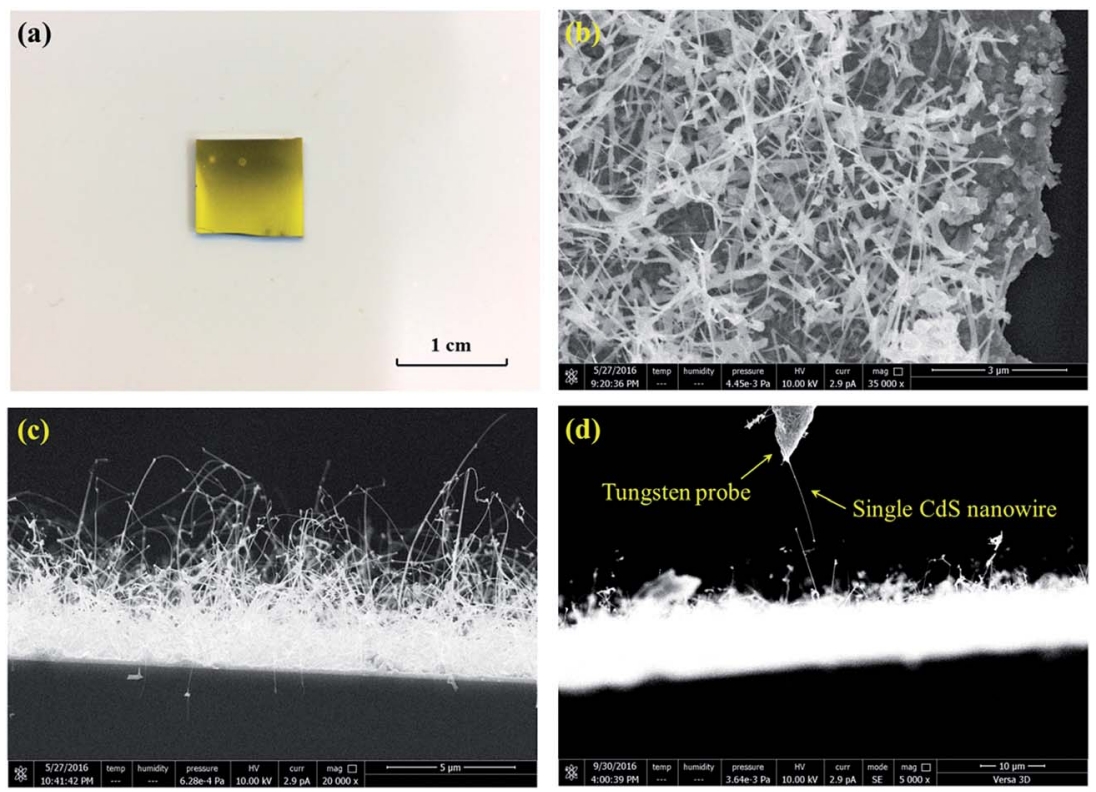

Fig. 1 Optical and SEM images of CdS nanowires. (a) Optical image of the synthesized CdS nanowire arrays on the substrate. (b) Top SEM image of CdS nanowire arrays. (c) Cross-sectional SEM image of CdS nanowire arrays. (d) An individual CdS nanowire picked up by a tungsten microprobe.
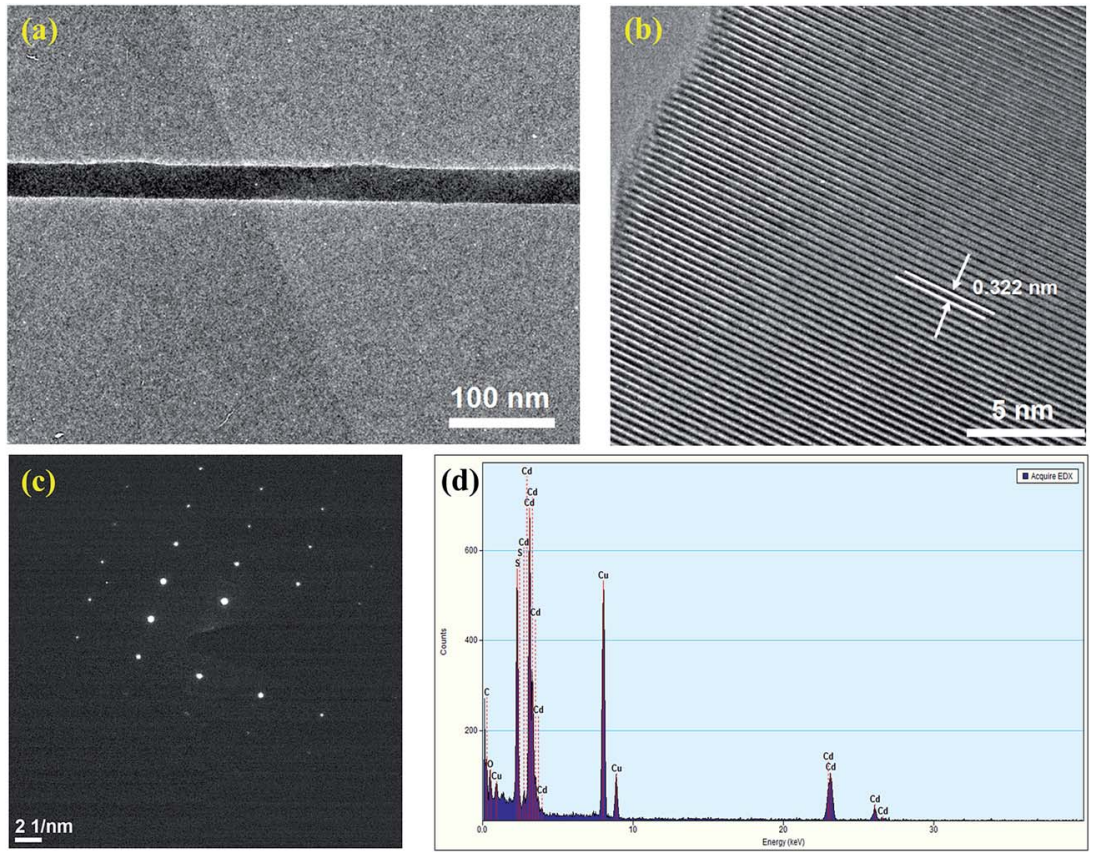

Fig. 2 High-resolution TEM image, SAED pattern, and EDX spectrum of a single CdS nanowire. (a) TEM image of a single separated CdS nanowire. (b) $0.322 \mathrm{~nm}$ lattice spacing displayed in the high-resolution TEM image. (c) Single-crystalline structure proved by SAED pattern. (d) Chemical characterization of the CdS nanowire by the EDX spectrum.

In the $\sigma$ measurement, the four-terminal sensing technique was applied to the suspended nanowire. A current $A_{\mathrm{s}}$ was fed to the nanowire sample through two small electrode pads in the middle (Fig. 3(c)) and two sensors were connected to the multimeter for measuring the voltage drop $V_{\mathrm{s}}$ across the sample. Then, the electrical conductivity was determined as $\sigma=4 A_{s} L /$
$\left(V_{\mathrm{s}} \pi d^{2}\right)$, where $L=3.05 \mu \mathrm{m}$ and $d=125 \mathrm{~nm}$ were respectively the length and diameter of the nanowire obtained from the SEM image.

In the $S$ measurement, one sensor was heated by an electrical current, and the temperature rise $\Delta T$ was measured through the resistance change of the sensor. Due to the temperature 

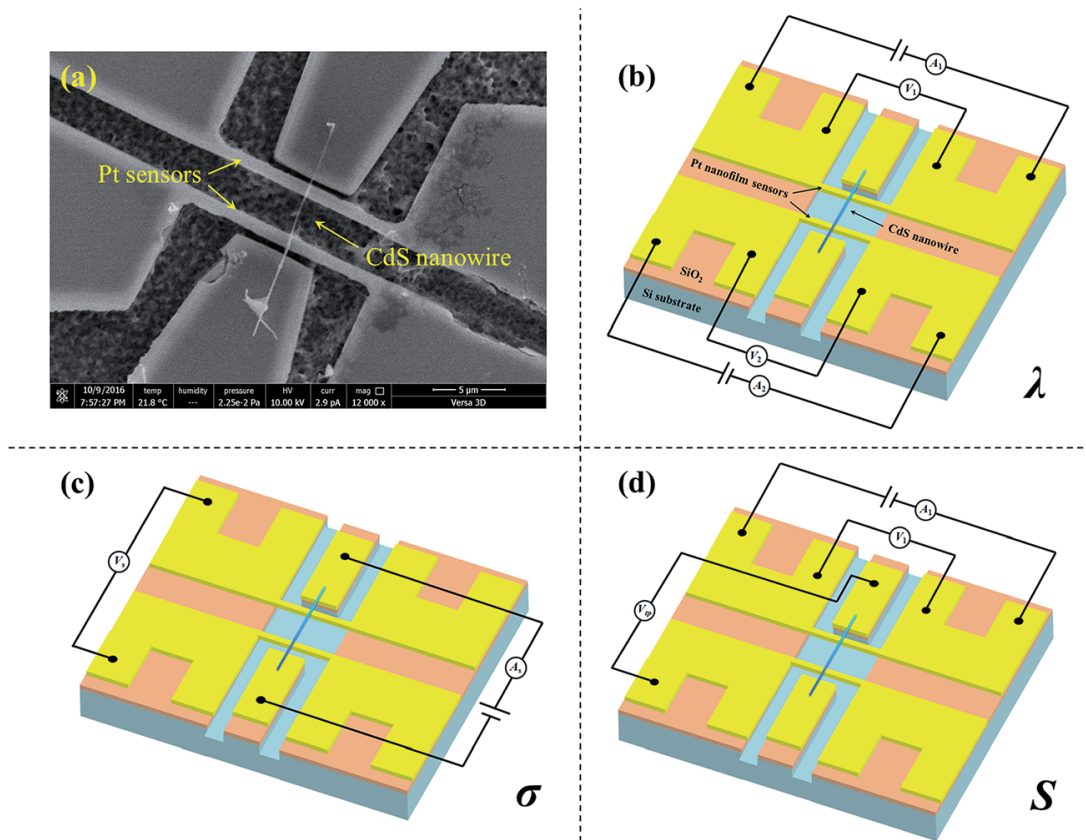

Fig. 3 SEM image of $\mathrm{H}$-type sensor with CdS nanowire. (a) Top view of suspended $\mathrm{H}$-type sensor and CdS nanowire. (b) Measurement of the thermal conductivity $\lambda . V_{1}, V_{2}, A_{1}$, and $A_{2}$ are the electrical voltages and currents of two sensors. (c) Measurement of the electrical conductivity $\sigma$. $V_{s}$ and $A_{s}$ are the voltage and current of the nanowire sample. (d) Measurement of the Seebeck coefficient $S$. $V_{\text {tp }}$ is the thermal potential of the nanowire sample.

difference across the nanowire, a thermoelectric potential $V_{\text {tp }}$ was generated in the CdS nanowire and was measured by a high-precision multimeter (Fig. 3(d)). The Seebeck coefficient was determined as $S=\left|V_{\mathrm{tp}} / \Delta T\right|$. All measurements were carried out in high vacuum $\left(<10^{-4} \mathrm{~Pa}\right)$. The heat losses from the nanowire and the sensor to the environment through the interactions with the rarefied gas or thermal radiation can be safely neglected. ${ }^{15}$ By simply changing the measurement schemes, all three thermoelectric parameters can be measured in situ on the same nanowire sample, greatly improving the accuracy and reliability of the experimental data. The detailed uncertainty analysis for the thermal conductivity, electrical conductivity and Seebeck coefficient is given in the ESI. $\dagger$

\section{Results and discussion}

Fig. 4 shows the results of the electrical measurement of CdS nanowire.

Our long-term goal is to fabricate efficient thermoelectric devices at room temperature. The temperature range of current work is from $0{ }^{\circ} \mathrm{C}$ to $46{ }^{\circ} \mathrm{C}$, with the temperature controlled using a Peltier heating/cooling stage installed inside the high vacuum chamber. As illustrated in Fig. 4, the current of nanowire has a linear relationship with the bias-voltage after $500 \mathrm{mV}$. The electrical resistance of nanowire was calculated from the $I^{-}$ $V$ curve from $500 \mathrm{mV}$ to $1000 \mathrm{mV}$. A negative temperature dependence of the resistivity of CdS nanowire is found in Fig. 4(c), which indicates that the CdS nanowire exhibits a typical semiconductor electrical behavior. The measured resistivity obeys the standard Arrhenius relation: ${ }^{15}$

$$
\rho(T)=\rho_{0} \exp \left(\frac{E_{0}}{k_{\mathrm{B}} T}\right),
$$

where $\rho$ is the resistivity, $k_{\mathrm{B}}$ is the Boltzmann constant, $E_{0}$ is the activation energy and $\rho_{0}$ is the pre-exponential factor. The results of linear fitting of the data to eqn (1) are shown in Fig. 4(c). The determined activation energy $E_{0}$ is $0.155 \mathrm{eV}$, less than one-tenth of the direct CdS band gap. $E_{0}$ represents the minimum amount of energy that is required to participate in the charge transport. The relatively small activation energy of the CdS nanowire means that more thermally excited charge carriers will contribute to the electrical conduction. These carriers play a dominant role in determining the electrical conductivity of the sample.

Based on the result shown in Fig. 4, the charge mobility of the CdS nanowire was calculated using the following formula: ${ }^{24}$

$$
\mu=\frac{1}{n e R}=\frac{1}{C V_{\mathrm{g}} R},
$$

where $\mu, n, e, R, C$, and $V_{\mathrm{g}}$ are the charge mobility, gate-induced carrier concentration, elementary charge, electrical resistance, gate capacitance and gate voltage, respectively. Here, the nanowire capacitance was estimated using a parallel plate capacitor model in vacuum, with $C=\varepsilon_{0} / \delta$, where $\varepsilon_{0}$ is the vacuum permittivity and $\delta$ is the distance between the nanowire and the silicon substrate. The calculated mobility increased with increasing temperature and reached the maximum value of $\sim 4000 \mathrm{~cm}^{2} \mathrm{~V}^{-1} \mathrm{~s}^{-1}$, which is one order of magnitude higher than the intrinsic mobility of CdS crystal platelet, 400 to $620 \mathrm{~cm}^{2} \mathrm{~V}^{-1}$ $\mathrm{s}^{-1} .{ }^{25}$ Such significant increment in charge mobility is due to the perfect single-crystalline structure of the nanowire (Fig. 2), where 

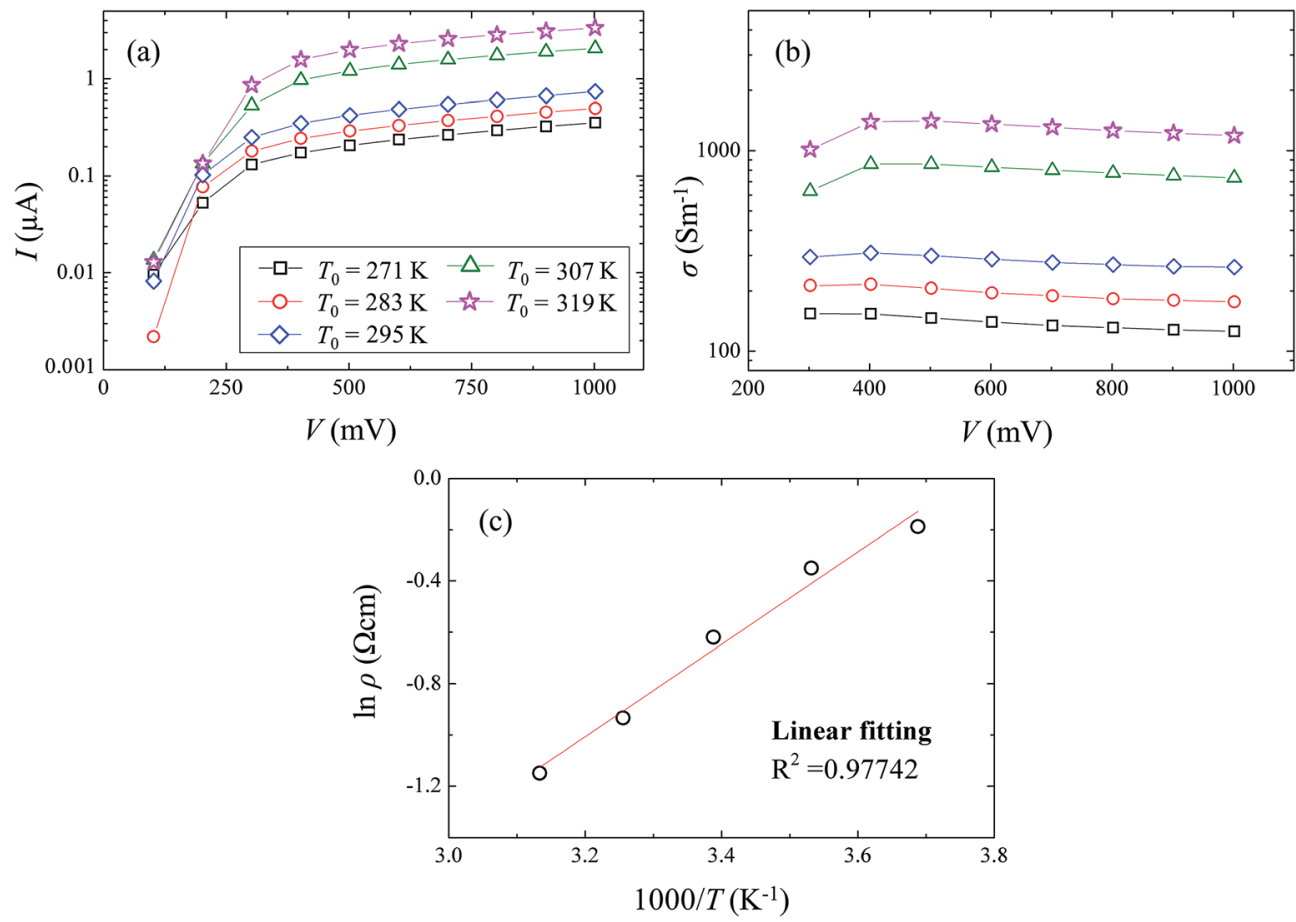

Fig. 4 Current vs. voltage characteristic curve (a) and electrical conductivity (b) of the CdS nanowire at different temperatures. (c) shows the linear relationship between $\ln (\rho)$ and the inverse temperature. The electrical measurements were carried out from 271 to $319 \mathrm{~K}$, where a Peltier heating/cooling stage was used to control the temperature. The results indicate that the electrical conductivity of the nanowire increases with increasing temperature.

the neutral and ionized impurities are minimized. ${ }^{26}$ In contrast to the other conventional thermoelectric materials, such as $\mathrm{Bi}_{2} \mathrm{~S}_{3}{ }^{15}$ the CdS nanowire has much higher electrical conductivity.

The measured thermal conductivities of the individual CdS nanowire and some other thermoelectric nanowires have been summarized in the Fig. 5. ${ }^{6,7,15-17,27,28}$ Our experimental result is in good agreement with the data measured using the optical Raman method (red line in Fig. 5). ${ }^{28}$ Due to the significant phonon-surface scattering effect, the thermal conductivity of CdS nanowire is only about one-tenth of that for the bulk material. ${ }^{29}$ With the rapid development of measurement techniques, ${ }^{30-32}$ this dramatic reduction in the thermal conductivity of the nanowire is commonly observed in the semiconductor nanowires. ${ }^{6,7,15}$ As illustrated in Fig. 5, the thermal conductivities of the different types of semiconductor nanowires are in the $0-5 \mathrm{~W} \mathrm{~m}{ }^{-1} \mathrm{~K}^{-1}$ range. For the nanowire with a smaller diameter, the thermal conductivity can be further suppressed. The thermal conductivity of the single-crystalline CdS nanowire is about twice as large as that of the $\mathrm{Bi}_{2} \mathrm{~S}_{3}$, Ge and $\mathrm{Bi}$ nanowires, probably due to its perfect lattice structure and smaller impurity content (see Fig. 2). For a thinner nanowire with a higher doping concentration, the thermal conductivity could be decreased further.

Fig. 6 shows the measured $Z T$ and Seebeck coefficient of the single-crystalline CdS nanowire. The Seebeck coefficient of the CdS nanowire is almost constant within the $260-330 \mathrm{~K}$ temperature range. The $Z T$ value of the CdS nanowire shows a significant increase with increasing temperature from $300 \mathrm{~K}$ to $320 \mathrm{~K}$. This rapid increase in $Z T$ is due to the significantly enhanced electrical conductivity. The CdS nanowire $Z T$ is around 0.003 at $300 \mathrm{~K}$, which is two orders of magnitude larger than that of the single-crystalline $\mathrm{Bi}_{2} \mathrm{~S}_{3}$ nanowire, ${ }^{15}$ and one order of magnitude smaller than that of the SnTe nanowire. ${ }^{6}$

Among three kinds of thermoelectric nanowires, $\mathrm{Bi}_{2} \mathrm{~S}_{3}$, CdS and SnTe, the electrical conductivity varies from $100 \mathrm{~S} \mathrm{~m}^{-1}$ of

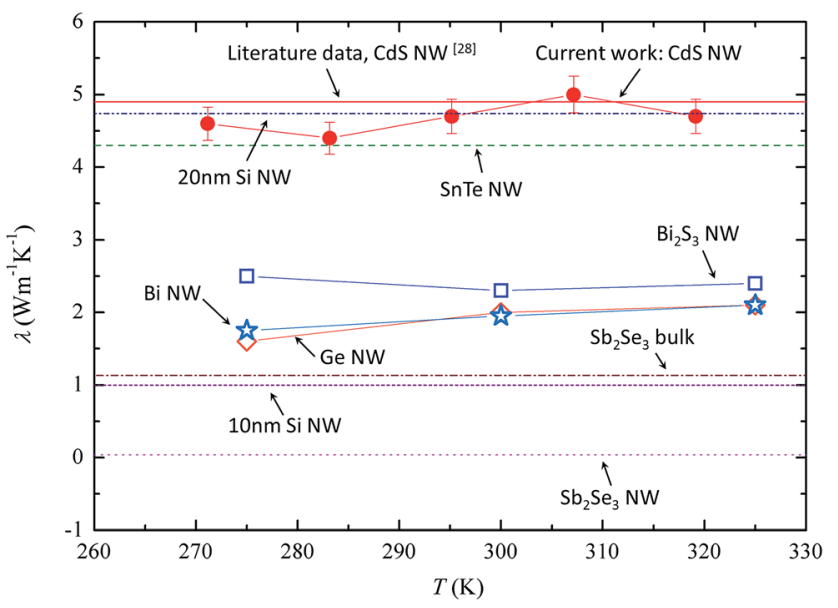

Fig. 5 Measured thermal conductivity of CdS nanowire. Red circles are the measured thermal conductivity values of the single-crystalline CdS nanowire with $5 \%$ uncertainty. The red line is the experimental data from literature. ${ }^{28}$ In addition, the data reported for some other thermoelectric nanowires are also shown in the figure: $\mathrm{Si}$ (NW) nanowire with 10 or $20 \mathrm{~nm}$ diameter, ${ }^{7}$ SnTe nanowire, ${ }^{6} \mathrm{Bi}_{2} \mathrm{~S}_{3}$ nanowire, ${ }^{15} \mathrm{Sb}_{2} \mathrm{Se}_{3}$ nanowire and bulk, ${ }^{27}$ Bi nanowire ${ }^{17}$ and Ge nanowire. ${ }^{16}$ 


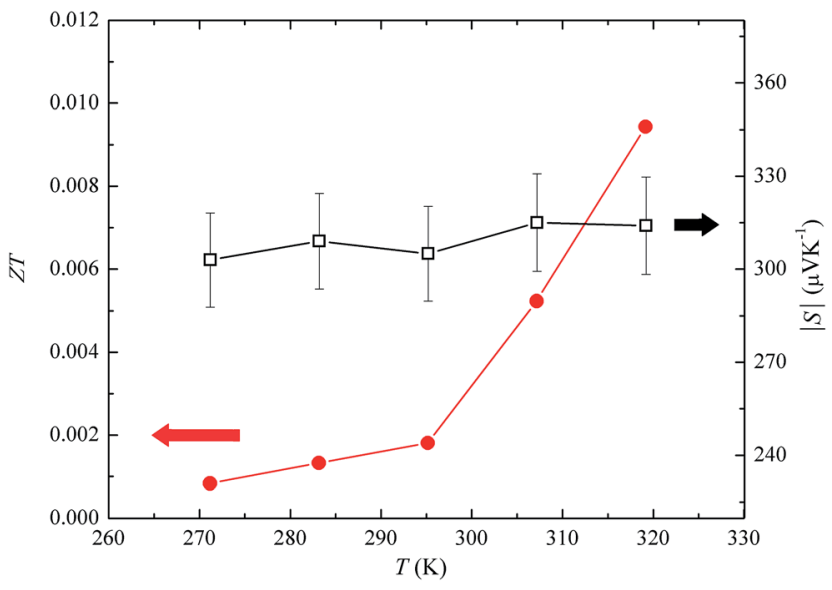

Fig. 6 Figure of merit and Seebeck coefficient of the individual singlecrystalline CdS nanowire as a function of temperature.

$\mathrm{Bi}_{2} \mathrm{~S}_{3}$ to $6 \times 10^{5} \mathrm{~S} \mathrm{~m}^{-1}$ of SnTe at room temperature. ${ }^{6}$ The thermal conductivity of nanowire varies from $2.3 \mathrm{~W} \mathrm{~m}^{-1} \mathrm{~K}^{-1}$ of $\mathrm{Bi}_{2} \mathrm{~S}_{3}$ to $4.7 \mathrm{~W} \mathrm{~m} \mathrm{~m}^{-1} \mathrm{~K}^{-1}$ of $\mathrm{CdS}$. The Seebeck coefficient varies from $50 \mu \mathrm{V} \mathrm{K}^{-1}$ of SnTe to $300 \mu \mathrm{V} \mathrm{K}^{-1}$ of CdS. It is clear that the electrical conductivity of nanowire plays the most important role in achieving high $Z T$ values. Doping the semiconductor nanowire with other atoms could be an efficient way to increase $Z T$ value, because the doped nanowire may have higher charge carrier concentration and lower thermal conductivity.

\section{Conclusions}

In this report, a novel H-type sensor was developed to measure the electrical conductivity, thermal conductivity and Seebeck coefficient of the same individual CdS nanowire. Compared to our previous T-type sensor or other microdevice methods, the H-type sensor method offers a high-precision four-terminal sensing measurement and ultra-high thermal sensitivity due to its suspended nanoscale structure. In this work, an individual nanowire was directly picked up from the arrays and connected to the sensor without contamination or damage caused by the conventional ultrasonic dispersion process. The CdS nanowire $Z T$ value was about 0.003 at room temperature, smaller than the values obtained for the SnTe or Si nanowires. This was mostly due to the limited electrical conductivity.

\section{Author contributions}

H. T. and X. Z. proposed and supervised the project. D. Z. and $\mathrm{W}$. H. prepared the CdS nanowire samples and provided high-quality TEM images. H. W. designed and performed the experiments. H. W. wrote the manuscript. All the authors checked and revised the manuscript.

\section{Acknowledgements}

The authors would like to thank Mr Tatsuya Ikuta, Dr Takanobu Fukunaga, Prof. Koji Takahashi, and Prof. Yasuyuki Takata for their help in fabricating the suspended H-type sensor and SEM operation. The work was supported by the JSPS KAKENHI Grantin-Aid for Young Scientists A No. $17 \mathrm{H} 04907$ and National Natural Science Foundation of China Grant No. 51636002, 51327001 and 51356001.

\section{References}

1 G. J. Snyder and E. S. Toberer, Complex thermoelectric materials, Nat. Mater., 2008, 7, 105.

2 J. R. Sootsman, D. Y. Chung and M. G. Kanatzidis, New and old concepts in thermoelectric materials, Angew. Chem., Int. Ed., 2009, 48, 86168639.

3 L. D. Zhao, S. H. Lo, Y. Zhang, H. Sun, G. Tan, C. Uher, C. Wolverton, V. P. Dravid and M. G. Kanatzidis, Ultralow thermal conductivity and high thermoelectric figure of merit in SnSe crystals, Nature, 2014, 508, 373.

4 J. F. Li, W. S. Liu, L. D. Zhao and M. Zhou, High-performance nanostructured thermoelectric materials, NPG Asia Mater., 2010, 2, 152.

$5 \mathrm{H}$. Bottner, G. Chen and R. Venkatasubramanian, Aspects of thin-film superlattice thermoelectric materials, devices, and applications, MRS Bull., 2006, 31, 211.

6 E. Z. Xu, Z. Li, J. A. Martinez, N. Sinitsyn, H. Htoon, N. Li, B. Swartzentruber, J. Hollingsworth, J. Wang and S. X. Zhang, Diameter dependent thermoelectric properties of individual SnTe nanowires, Nanoscale, 2015, 7, 2869.

7 A. I. Boukai, Y. Bunimovich, J. Tahir-Kheli, J. K. Yu, W. A. Goddard and J. R. Heath, Silicon nanowires as efficient thermoelectric materials, Science, 2008, 451, 168.

8 A. I. Hochbaum, R. Chen, R. D. Delgado, W. Liang, E. C. Garnett, M. Najarian, A. Majumdar and P. Yang, Enhanced thermoelectric performance of rough silicon nanowires, Nature, 2008, 451, 163.

9 R. Venkatasubramanian, E. Siivola, T. Colpitts and B. O'Quinn, Thin-film thermoelectric devices with high room-temperature figures of merit, Nature, 2001, 413, 597.

10 B. Poudel, Q. Hao, Y. Ma, Y. Lan, A. Minnich, B. Yu, X. Yan, D. Wang, A. Muto, D. Vashaee, X. Chen, J. Liu, M. S. Dresselhaus, G. Chen and Z. Ren, HighThermoelectric performance of nanostructured bismuth antimony telluride bulk alloys, Science, 2008, 320, 634.

11 T. C. Harman, P. J. Taylor, M. P. Walsh and B. E. LaForge, Quantum dot superlattice thermoelectric materials and devices, Science, 2002, 297, 2229.

12 L. D. Hicks and M. S. Dresselhaus, Thermoelectric figure of merit of a one dimensional conductor, Phys. Rev. B: Condens. Matter Mater. Phys., 1993, 47, 16631.

13 G. D. Mahan and J. O. Sofo, The best thermoelectric, Proc. Natl. Acad. Sci. U. S. A., 1996, 93, 7436.

14 T. E. Humphrey and H. Linke, Reversible thermoelectric nanomaterials, Phys. Rev. Lett., 2005, 94, 096601.

15 W. G. Ma, T. T. Miao, X. Zhang, K. Takahashi, T. Ikuta, B. P. Zhang and Z. H. Ge, A T-type method for characterization of the thermoelectric performance of an individual free-standing single crystal $\mathrm{Bi}_{2} \mathrm{~S}_{3}$ nanowire, Nanoscale, 2016, 8, 2704. 
16 M. C. Wingert, Z. C. Y. Chen, E. Dechaumphai, J. Y. Moon, J. H. Kim, J. Xiang and R. K. Chen, Thermal conductivity of $\mathrm{Ge}$ and $\mathrm{Ge}-\mathrm{Si}$ core-shell nanowires in the phonon confinement regime, Nano Lett., 2011, 11, 5507.

17 J. W. Roh, K. Hippalgaonkar, J. H. Ham, R. K. Chen, M. Z. Li, P. Ercius, A. Majumdar, W. C. Kim and W. Y. Lee, Observation of anisotropy in thermal conductivity of individual single-crystalline bismuth nanowires, ACS Nano, 2011, 5, 3954.

18 M. Y. Zhang, M. Wille, R. Röder, S. Heedt, L. B. Huang, Z. Zhu, S. Geburt, D. Grutzmacher, T. Schäpers, C. Ronning and J. G. Lu, Amphoteric nature of Sn in CdS nanowires, Nano Lett., 2014, 14, 518.

19 Y. Zhao, X. C. Yang, W. H. Huang, X. Zou and Z. G. Lu, Synthesis and optical properties of CdS nanowires by a simple chemical deposition, J. Mater. Sci., 2010, 45, 1803.

20 G. Y. Gou, G. Z. Dai, C. Qian, Y. F. Liu, Y. Fu, Z. Y. Tian, Y. K. He, L. G. Kong, J. L. Yang, J. Sun and Y. L. Gao, Highperformance ultraviolet photodetectors based on CdS/CdS: $\mathrm{SnS}_{2}$ superlattice nanowires, Nanoscale, 2016, 8, 14580.

21 D. S. Zheng, H. H. Fang, P. Wang, W. J. Luo, F. Gong, J. C. Ho, X. S. Chen, W. Lu, L. Liao, J. L. Wang and W. D. Hu, Highperformance ferroelectric polymer side-gated CdS nanowire ultraviolet photodetectors, Adv. Funct. Mater, 2016, 26, 7690.

22 K. Nishimura, H. D. Wang, T. Fukunaga, K. Kurata and H. Takamatsu, Measurement of in-plane thermal and electrical conductivities of thin film using a micro-beam sensor: A feasibility study using gold film, Int. J. Heat Mass Transfer, 2016, 95, 727.

23 H. D. Wang, S. Q. Hu, K. Takahashi, X. Zhang, H. Takamatsu and J. Chen, Experimental study of thermal rectification in suspended monolayer graphene, Nat. Commun., accepted.
24 H. D. Wang, X. Zhang and H. Takamatsu, Ultraclean suspended monolayer graphene achieved by in situ current annealing, Nanotechnology, 2016, 28, 045706.

$25 \mathrm{~K}$. W. Boer and K. Bogus, Electron mobility in CdS at high electric fields, Phys. Rev., 1968, 176, 899.

26 X. C. Yang, C. C. Xu and N. C. Giles, Intrinsic electron mobilities in CdSe, CdS, ZnO, and $\mathrm{ZnS}$ and their use in analysis of temperature-dependent Hall measurements, $J$. Appl. Phys., 2008, 104, 073727.

27 T. Y. Ko, M. Shellaiah and K. W. Sun, Thermal and thermoelectric transport in highly resistive single $\mathrm{Sb}_{2} \mathrm{Se}_{3}$ nanowires and nanowire bundles, Sci. Rep., 2016, 6, 35086.

28 X. F. Liu, R. Wang, Y. P. Jiang, Q. Zhang, X. Y. Shan and X. H. Qiu, Thermal conductivity measurement of individual CdS nanowires using microphotoluminescence spectroscopy, J. Appl. Phys., 2010, 108, 054310.

29 Cadmium Sulfide (CdS) semiconductors, Database from AZO Materials, 2013, http://www.azom.com/article.aspx? ArticleID $=8407$.

30 J. H. Liu, H. D. Wang, W. G. Ma, X. Zhang and Y. Song, Simultaneous measurement of thermal conductivity and thermal contact resistance of individual carbon fibers using Raman spectroscopy, Rev. Sci. Instrum., 2013, 84, 044901.

31 H. D. Wang, K. Kurata, T. Fukunaga, H. Takamatsu, X. Zhang, T. Ikuta, K. Takahashi, T. Nishiyama, H. Ago and Y. Takata, In-situ measurement of the heat transport in defect-engineered free-standing single-layer graphene, Sci. Rep., 2016, 6, 21823.

32 H. D. Wang, J. H. Liu, Z. Y. Guo, X. Zhang, R. F. Zhang, F. Wei and T. Y. Li, Thermal transport across the interface between a suspended single-walled carbon nanotube and air, Nanoscale Microscale Thermophys. Eng., 2013, 17, 349. 